\title{
Production and Properties Analysis of Honey Nanofibers Enriched with Antibacterial Herbal Extracts for Repair and Regeneration of Skin and Bone Tissues
}

\author{
Esmaeil Shahbazi ${ }^{1}$ and Kiumars Bahrami ${ }^{1,2}$ \\ 1. Department of Organic Chemistry, Faculty of Chemistry, Razi University, Kermanshah 67149-67346, Iran \\ 2. Nanoscience and Nanotechnology Research Center (NNRC), Razi University, Kermanshah 67149-67346, Iran
}

\begin{abstract}
Among the commonly used nanofibers production methods, electrospinning has many advantages such as ease of production, possibility of industrialization, nanofibers dimensional control and repeatability. Many parameters affect the characteristics of the nanofibers produced by this method, the most important of these parameters being the applied voltage, the concentration of polymer solution, the sample injection rate, the distance between the needle and the collector, and environmental factors too. Pharmaceutical properties of nanofibers are determined by their composition and structure at the nanoscale. Therefore, the ultimate goal of identify nanostructure and nanofiber morphology must be searching for an atom to an atom on a surface and under the reaction conditions. In this paper, honey nanofibers enriched with antibacterial herbal extracts such as the garlic, mint and edible mushroom are produced by method of electrospinning, by reviewing the effective conditions in preparing them, to achieve nanostructures and optimize conditions. The related nanofibers have ability to repair and regenerate damaged skin and bone tissues as an effective drug, which is especially important for biocompatibility and economics. In this research, nanofibers have been investigated by examining cases affecting structure and performance. The hydroalcoholic extracts and nanofibers are identified by device methods such as GC-Mass (gas chromatography-mass spectrometry), FT-IR (Fourier transform infrared spectroscopy), SEM (scanning electron microscopy) and XRD (X-ray diffraction spectroscopy).
\end{abstract}

Key words: Natural antibiotic, honey nanofibers, Allium sativum, skin and bone tissues, antibacterial properties, regeneration.
Highlights
- $\quad$ Among the nanofibers production methods, electrospinning has many advantages.
- Many parameters affect the characteristics of the nanofibers produced.
- Medicinal properties of nanofibers are determined by their composition and structure.
- Antibacterial herbal extracts increase pharmaceutical properties of honey.
- $\quad$ Nanofibers have the ability to repair and regenerate damaged skin and bone tissues.

\section{Introduction}

Due to the increasing resistance of pathogenic bacteria to antibiotics, researchers are seeking new herbal medicines as alternatives to chemical drugs and antibiotics [1]. In addition to antimicrobial and antiseptic properties, medicinal plants such as garlic, mint an mushrooms have therapeutic properties for

Corresponding author: Kiumars Bahrami, kbahrami2@hotmail.com, Ph.D., professor, research fields: applications of nanostructured materials for the synthesis of organic compounds. skin and bone diseases, as well as the ability to reconstruct and repair these tissues [2]. By extracting these plants and combining it with honey polymer material, which has the same properties for the treatment and repair of broken tissues of the body, and by producing an electrospinning device with a nanoscale, this nanofiber can be used as a medicine in tissue engineering, drug delivery and wound healing [3-6].

Garlic with scientific name Allium sativum L. is a natural antibiotic, antifungal and strong antivirus that is 
rich in folic acid, vitamin $\mathrm{C}$, calcium, iron, magnesium, potassium, and zinc and vitamins $\mathrm{B}_{1}, \mathrm{~B}_{2}$ and $\mathrm{B}_{3}$. Garlic is used as an anticoagulant and as a natural drug for dilution of blood [7-9]. Garlic is also effective in increasing white blood cells counts [10]. Strong antioxidants in garlic neutralize free radicals that cause aging and strengthen the immune system [11].

Mint with scientific name Mentha spicata L., has antibacterial, antifungal, anticancer, antifungal and antioxidant properties [12, 13]. Mint extracts and oil are a beneficial for skin. This material regulates skin secretions and makes skin clear and clean in people with oily skin $[14,15]$.

Mushroom with scientific name Agaricus bisporus, plays an important role in the elimination of viral, bacterial, fungal, and fungal infections and the resistance of viruses, bacteria and fungi to common antibiotics due to their various polysaccharide compounds $[16,17]$. The mushrooms contain vitamins B, C, D and antioxidants. Therapeutic properties of mushroom include anti-inflammatory, anti-cancer and anti-aging properties [18]. It is a strong antioxidant and natural moisturizer. Therefore, its extract is used as an active ingredient in topical creams, serums and facial products today [19].

Honey is known as a natural substance with the most potent antibacterial property. This material is the simplest and most studied activity and antibacterial and disinfectant effect. Its antibacterial activity is usually attributed to high sugar content and acidity [20]. It is used as a conventional treatment for wounds, burns and wounds disinfection. United States National Honey Association, because it contains a small amount of different types of vitamins and minerals (niacin, riboflavin, pantothenic acid, aluminum, calcium, chromium, chlorine, copper, iron, magnesium, manganese, phosphorus, potassium, selenium, silica, sodium, sulfur and zinc), it is highly recommended for honey [21].

When a drug is mixed with honey, it is quickly and easily absorbed by the body, and the whole body system and blood circulation are effective. In the meantime, honey preserves its medicinal properties and its effects remain for a long time [22].

Honey is a natural polymer, due to its biocompatibility, biodegradability and non-toxicity. It has the potential to be used in various fields, including medical and pharmaceutical industries. The herbal nanofibers are prepared by a combination of drug extraction and electrospinning, and will be studied under different operating conditions [23]. Electrospinning nanofibers are used with a unique structure as a three-dimensional grid for tissue engineering and scaffolds for tissue growth. Polymer nanofibers have a wide range of applications due to properties such as delicacy and surface to volume ratio, high porosity, three-dimensional grid capability and having favourable mechanical and biological properties. By locating these herbal nanomaterials on wounds and damaged tissues, they can be regenerated to a considerable extent. Because of providing a location for adhesion, increased contact surface, support for large cell mass and the ability to form a special structure, porous scaffolding has special importance in textiles engineering. Polymer matrices are made of natural and synthetic polymers to create a structure similar to the natural extracellular matrix (ECM) [24].

In this research work, the production and properties analysis of honey nanofibers enriched with antibacterial herbal extracts for repair and regeneration of skin and bone damaged tissues were discussed.

\section{Experimental}

\subsection{Preparation of Hydroalcoholic Extracts}

Initially, garlic, fresh mint and edible mushroom were prepared. Then, the young and healthy plants were isolated. Extraction of hydroalcoholic extract of dried and crushed plants using continuous extraction method was carried out with Soxhlet system and heat at boiling point of ethanol solvent. For this purpose, about $200 \mathrm{~g}$ of powder of the desired plants were weighed by 
digital scales of the Sartorius cp224s model with accuracy of $0.1 \mathrm{mg}$, and poured in a cylindrical cartouche made of Watman filter paper (Watmann 0.5 $\mathrm{mm}$, USA, SAMFORD), and was located in a one-liter Soxhlet extractor. Laboratory water bath temperature was set at $70{ }^{\circ} \mathrm{C}$ under extraction conditions with the solvent mentioned. The extraction operation continued until the extract was colorless in the soxhlet. The extracts were dried with a vacuum rotary evaporator (Heidolph WD 2000, Brinkmann, Canada) at $40{ }^{\circ} \mathrm{C}$. Then, they were stored in glass containers in the refrigerator until they were used.

\subsection{Method of Chemical Compounds Analysis of Extracts}

Since the compounds in the extract are known in terms of molecular weight and polarity as volatile substances, the analysis of the chemical compounds of the extract of the tested plants was carried out by a gas chromatography coupled to mass spectrometry (Agilent 5975 C type, made in USA). To determine the type of bonds present in the sample, analysis was performed by FT-IR spectrometer (Bruker, Alpha).

\subsection{Production of Nanofibers}

Electrospinning is a common, simple and effective method for the production of separated polymeric nanofibers, in which only a small amount of polymer is needed to produce nanofibers. The basis of the electrospinning process is based on the elongation of the polymer fluid. The equipment used for this technique includes high voltage power supply, pump, spinner and collector. The properties of the formed nanofibers depend on molecular weight, surface tension, viscosity, conductivity of solution, dielectric constant of solvent, potential difference, the distance between the syringe tip (the nozzle head) and the wheel (collector), feed rate, temperature, effect of collector, needle diameter, moisture content and environmental factors.

\subsection{Steps to Doing Research Work}

In this paper, honey nanofibers enriched with antibacterial herbal extracts have been studied for repair and regeneration of skin and bone tissues with the help of the electrospinning process. Firstly, the extracts should be prepared by a soxhlet extraction method. In order to prepare a polymeric solution, various amounts of honey as a biodegradable polymer were dissolved in ethanol. Then, equal volume ratios of the desired extracts were added to the polymer solution (70: 10: 10:10) and located in room temperature under the stirrer at $300 \mathrm{rpm}$ for 1 hour. The speed and mode of mixing is very important in preparing the solution, because the solution will be smooth and homogeneous.

Finally, the obtained mixtures were located under different conditions of electrospinning by laboratory machine Three Side Lab ES model, manufacture in Nanoazma Company. For the production of nanofibers, different conditions were compared with each other, and the best conditions were obtained for producing the coating on the nanoscale and also the identical.

During the electrospinning process, the final polymeric solutions with different viscosity were poured separately into a $5 \mathrm{~mL}$ plastic syringe with an internal diameter of $13 \mathrm{~mm}$. The syringe was connected to a stainless steel needle with a diameter of $0.7 \mathrm{~mm}$ (gauge 18) with a smooth tip, by connector (silicone hose). The air inside the syringe and needle was removed by pressing the solution, completely.

The needle tip is connected to the positive pole and the collector plate is connected to the negative pole instead of the earthing system. In order to control the flow of polymeric solution and having a stable and constant polymer flow, the syringe was placed on a syringe driver. This pump moves back and forth at speeds of $20 \mathrm{~mm} / \mathrm{s}$.

The electrospinning process was carried out at an injection rate (outflow rate) in the range of 0.3-0.8 $\mathrm{mL} / \mathrm{hr}$. The applied voltage between the needle tip and the collector drum coated with an aluminum sheet was under a potential difference in the range of $10-25 \mathrm{kV}$. The polymer solution was sent to a rotating aluminum cylinder as a nanofibers collector (with a diameter of 8 
$\mathrm{cm}$ and a length of $30 \mathrm{~cm}$ ), that it was located $10 \mathrm{~cm}$ from the needle tip. In order to leave the solvent and residual water, the electrospun samples were located in room temperature for one day to dry completely. All experiments were performed in a lab environment at a temperature of about $25^{\circ} \mathrm{C}$, and the polymer solutions were used after preparation in the electrospinning process, immediately. In order to carry out electrospinning process tests, to achieve the appropriate thickness of the nanofibers scaffolds that can be removed from the aluminum foil, the electrospinning process was performed for 6 hours and was used from circular collector plate at $400 \mathrm{rpm}$. Finally, the morphology and diameter of the electrospun nanofibers were studied using the Scanning Electron Microscope (S-360 Oxford Eng. model), and analyzed using image analysis software (ImageJ 1.44p). The optimal sample was analyzed by $\mathrm{X}$-Ray Diffraction machine (Inel-model, France).

If nanofibrous scaffolds containing the cultured cells transplanted into the patient's body (on the wounded tissue), they will repair damaged tissues. In fact, from the perspective of biological, body membranes such as blood vessels, cartilage, skeletal system, nerve and skin are similar to electrospun nanofibers. In order to investigate the stability of the nanofibers scaffold in the body tissue, scaffolds of the nanofibers containing the extract were immersed in acetate buffer for 24 hours at $37{ }^{\circ} \mathrm{C}$. Then, the removed samples from the solution were located at room temperature for 24 hours to dry completely. After that, changes in the structure of nanofibers scaffolds were investigated by SEM electron microscope before and after than located in acetate buffer solution.

\section{Results and Discussion}

\subsection{Determine the Type and Amount of Identified Compounds from the Extracts}

The GC-Mass results were analyzed using various sources, and IUPAC and the common names of the chemical compounds identified were presented in the original interpretation. In garlic extract, compounds containing diallyl disulfide (25.27\%), trisulfide, di-2-propenyl (20.01\%), diallyl tetrasulfide (18.45\%), methyl allyl trisulfide (13.02\%), nitrosothymol (7.04\%) and diallyl sulfide $(5.17 \%)$ were analyzed as the main components of the substance. The total compounds detected from garlic extract with using GC-Mass analysis are shown in Table 1.

Differences in the obtained chemical compounds from extracts by different researchers can be attributed to changes in weather conditions, harvest time, type of fertilizer used, position and geographical location of cultivation of crops and other influences [25, 26].

The extract of this plant contains sulfur compounds that fight cancer. It strengthens bone tissue, also maintains the health of blood vessels. Garlic helps to prevent the formation of blood clots by increasing fibrinolytic activity, increasing coagulation

Table 1 The compounds identified from garlic extract using gas chromatography-mass spectrometry (GC-MS).

\begin{tabular}{llll}
\hline Number & Retention time $(\mathrm{min})$ & Compound & Percentage $(\%)$ \\
\hline 1 & 4.557 & 1,2 -Dithiolane & 0.54 \\
2 & 4.866 & Allyl sulphide & 2.14 \\
3 & 12.065 & $1,3,5$-Trithiane & 3.21 \\
4 & 13.896 & Diallyl sulfide & 5.17 \\
5 & 14.514 & Methyl allyl trisulfide & 13.02 \\
6 & 18.685 & Diallyl disulfide & 25.27 \\
7 & 20.167 & Trisulfide, di-2-Propenyl (Diallyl trisulfide) & 20.01 \\
8 & 22.774 & Diallyl tetrasulfide & 18.45 \\
9 & 25.094 & Silane, butyltrimethoxy- & 3.17 \\
10 & 28.870 & Nitrosothymol & 7.04 \\
\hline Total & & & 98.02 \\
\hline
\end{tabular}


and flocculation and platelet aggregation [27]. The structural formulas of the obtained main chemical compounds from the garlic extract with using GC-Mass analysis are shown in Fig. 1.

In mint extract, compounds containing Menthol (43.31\%), Mentone (19.43\%), Menthyl acetate (9.78\%), Neomenthol (6.13\%) and 1,8-Cineole (5.81\%) were analyzed as the main components of the substance. The total compounds detected from mint extract with using GC-Mass analysis are shown in Table 2.

The main ingredients of mint extract are including menthol and mentone. Menthol stimulates the senses of cold feel in the skin, selectively. This material blocks the sensitive channels of sodium, and stimulates the muscles by reducing their activity. Also, taking it on the skin causes the vessels to dilate and creates a feeling of coldness, which leads to the appearance of an analgesic effect by inducing local anesthesia. Mentone is a monoterpene with spicy taste that exists in a number of essential oils, naturally. The mentone structure is similar to menthol, that there is a secondary alcohol functional group instead of the carbonyl functional group [28]. The structural formula of the obtained main chemical compounds from the mint extract with using GC-Mass analysis is shown in Fig. 2.

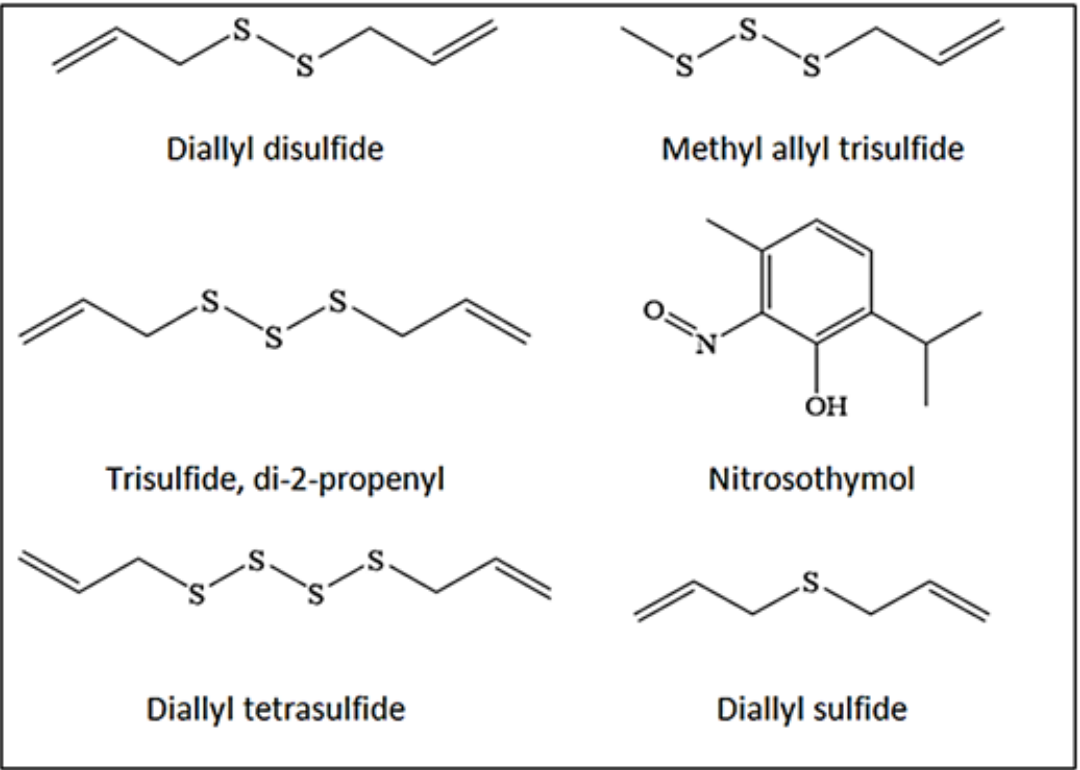

Fig. 1 The structural formula of the main compounds obtained from the garlic extract by GC-Mass.

Table 2 The compounds identified from mint extract using gas chromatography-mass spectrometry (GC-MS).

\begin{tabular}{llll}
\hline Number & Retention time $(\mathrm{min})$ & Components & Percentage $(\%)$ \\
\hline 1 & 6.19 & Beta-pinene & 0.60 \\
2 & 7.35 & Limonene & 4.01 \\
3 & 7.93 & 1,8 -Cineole & 5.81 \\
4 & 10.85 & Mentone & 19.43 \\
5 & 11.03 & Neomenthol & 6.13 \\
6 & 11.17 & Menthol & 43.31 \\
7 & 13.45 & Menthyl acetate & 9.78 \\
8 & 15.97 & Trance-beta- caryophyllene & 2.27 \\
9 & 17.13 & Germacrene-d & 3.16 \\
10 & 19.75 & Viridiflorol & 2.60 \\
\hline Total & & & 97.01 \\
\hline
\end{tabular}




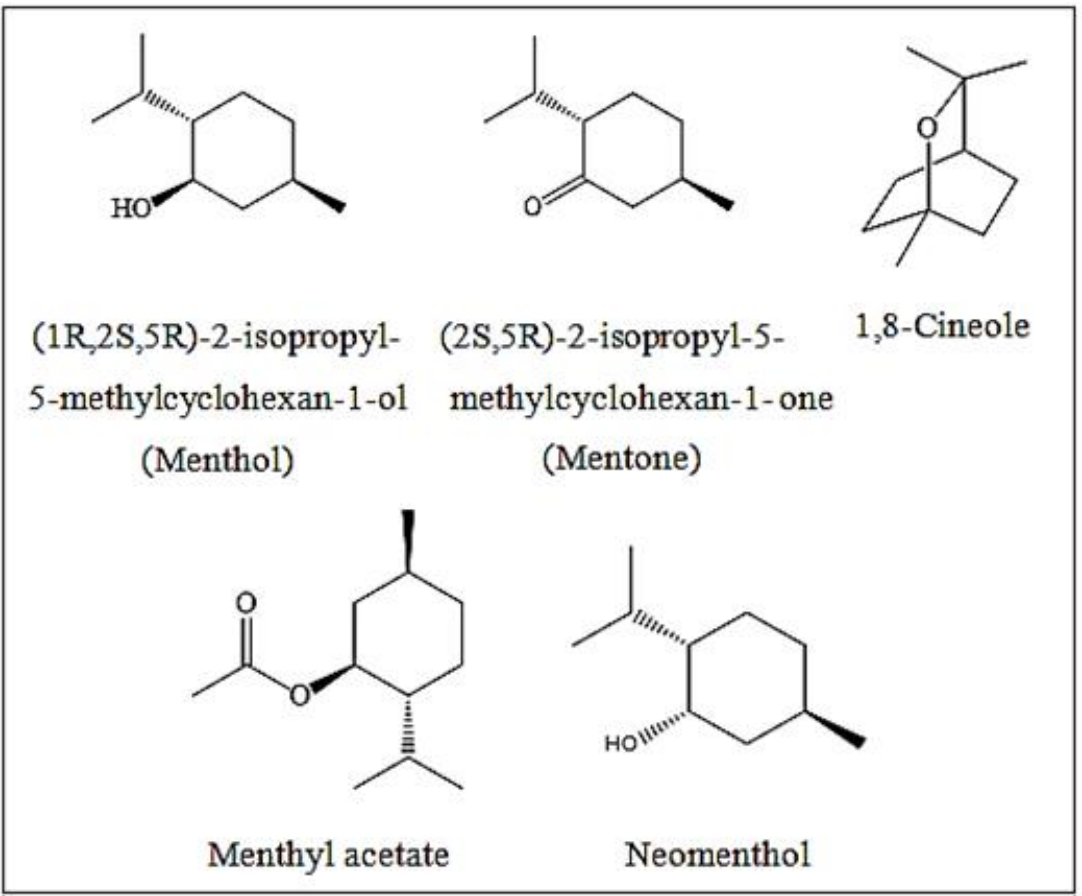

Fig. 2 The structural formula of the main compounds obtained from the mint extract by GC-Mass.

Table 3 The compounds identified from the edible mushroom extract using gas chromatography-mass spectrometry (GC-MS).

\begin{tabular}{llll}
\hline Number & Retention time (min) & Components & Percentage (\%) \\
\hline 1 & 3.01 & 1,3,2-Dioxaborolan-4-one, 2-ethyl-5-methyl- & 0.4 \\
2 & 12.50 & Butyl aldoxime, 3-methyl-, anti & 0.2 \\
3 & 14.19 & Pentanal, oxime & 2.13 \\
4 & 14.51 & Butanal, oxime & 6.41 \\
5 & 17.04 & Hexane-2,6-di(isonitrile), 1-(formyloxymethyl)_ & 9.17 \\
6 & 18.77 & 2-Norbornanone & 32.96 \\
7 & 19.09 & 6-Oxabicyclo[3.1.0]hexane-3-carbonitrile & 7.35 \\
8 & 23.27 & Methanamine, N-pentylidene- & 9.14 \\
9 & 29.50 & 1,4-Methanobenzocyclodecene, tetradecahydro- & 25.09 \\
10 & 32.23 & 1H-Purine-2,6-dione, & 3,7-dihydro-1,3-dimethyl-7-[2-[(1-methyl-2-phenylethyl)amino] ethyl]- \\
& & [Fenethylline] & 9.61 \\
\hline Total & & & 98.46 \\
\hline
\end{tabular}

In mushroom extract, compounds containing 2-norbornanone

(32.96\%),

1,4-methanobenzocyclodecene, tetradecahydro(25.09\%), hexane-2,6-di(isonitrile), 1-(formyloxymethyl) (9.17\%), methanamine, $\mathrm{N}$-pentylidene (9.14\%), 6-oxabicyclo[3.1.0]hexane-3-carbonitrile (7.35\%) and butanal, oxime $(6.41 \%)$ were analyzed as the main components of the substance. The total compounds detected from mushroom extract with using GC-Mass analysis are shown in Table 3.

The edible mushrooms have low energy content and do not have cholesterol, fat and gluten. Their sodium levels are low, but instead they contain large amounts of selenium, potassium, riboflavin, niacin and vitamin D. Mushrooms contain lectins, which fight cancer cells, and prevent their proliferation. Also, mushrooms have polysaccharides that boost the immune system [29]. The structural formula of the obtained main chemical compounds from the edible mushroom extract with 
using GC-Mass analysis is shown in Fig. 3.

\subsection{Analysis and Interpretation of the FT-IR Spectrum}

of Obtained Solid Mass from the Evaporation of Extracts

Infrared spectroscopy is based on absorption of radiation and the study of the vibrational mutations of molecules and multi-atomic ions. This method is used as a powerful and developed method for determining the structure and measurement of chemical species. The interaction of infrared radiation with a sample causes changes in the vibration energy of bond in its molecules, and it is a suitable method for identification of functional groups and molecular structures (Figs. 4 to 6 and Tables 4 to 6 ).

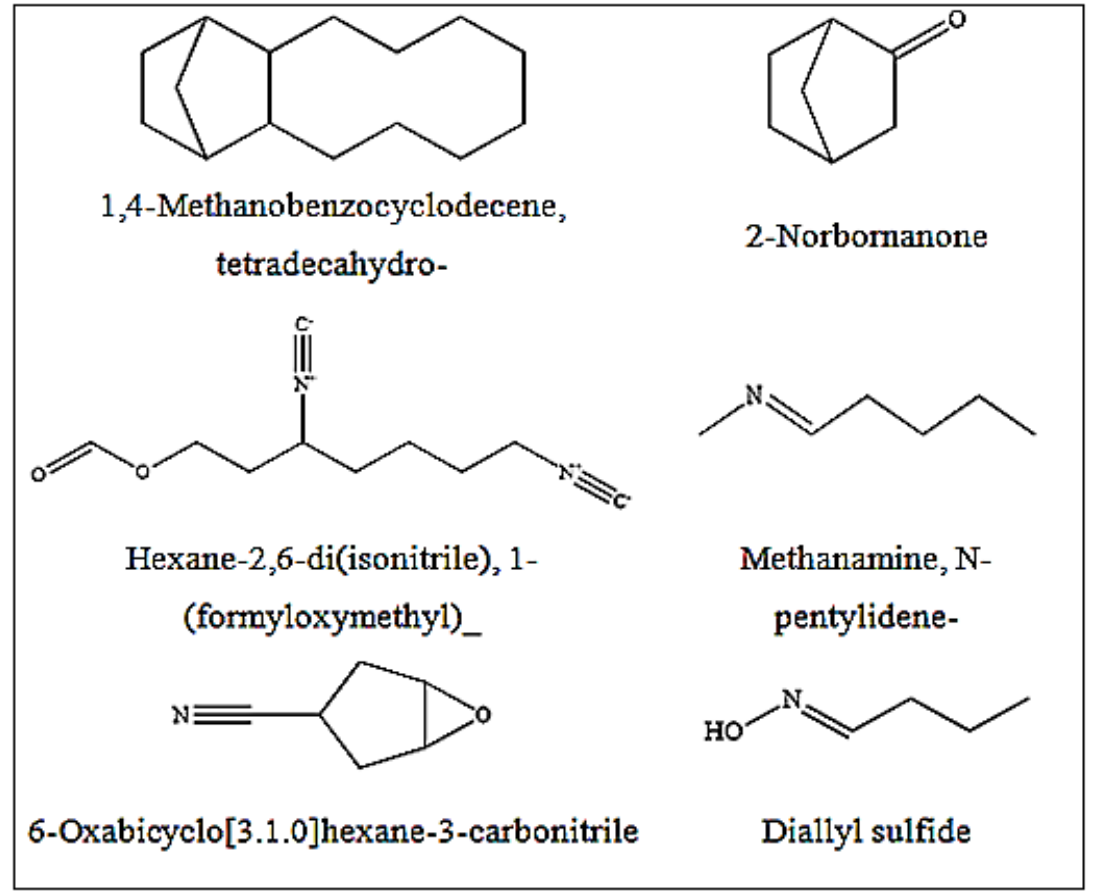

Fig. 3 The structural formula of the main compounds obtained from the edible mushroom extract by GC-Mass.

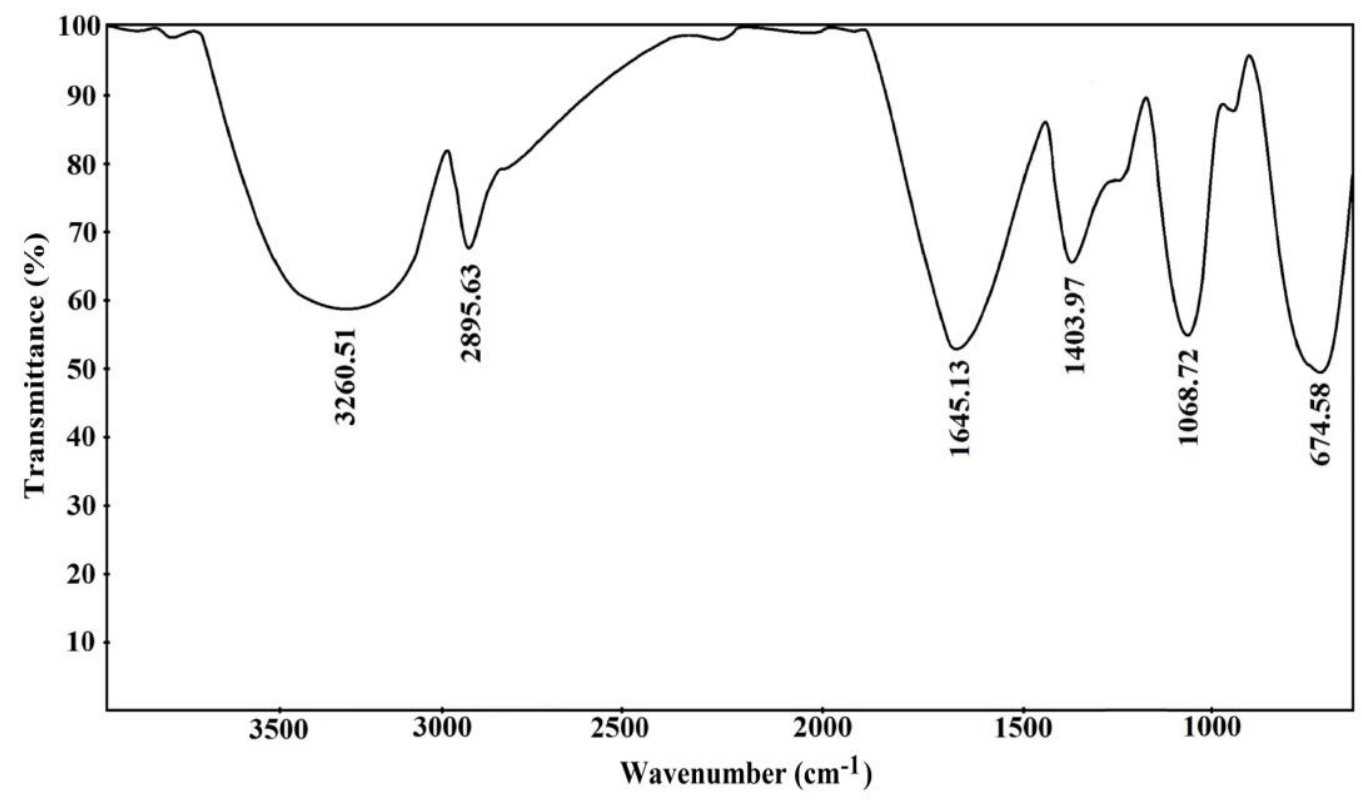

Fig. 4 FT-IR spectrum of solid mass obtained from evaporation of garlic extract. 
Table 4 Analysis of functional group, vibrational transitions and type of bonds found in the garlic extract (Fourier transform infrared spectroscopy).

\begin{tabular}{lllll}
\hline Frequency $\left(\mathrm{cm}^{-1}\right)$ & Bond & Functional group & Type of bond & Specific type of bond \\
\hline $3,260.51$ & O-H & Hydroxyl & Broad, stretch & $\begin{array}{l}\text { Polyhydroxy compounds such as flavonoids, } \\
\text { non-flavonoids and saponins }\end{array}$ \\
$2,895.63$ & C-H & Aromatic & Asymmetric stretch & Aromatic compounds \\
$1,645.13$ & C=O & Carboxyl, Carbonyl & Stretch & Peptide bond \\
$1,403.97$ & O-H & Carboxyl & Bending & Flavonoids, tannins, saponins and glycosides \\
$1,068.72$ & S=O & Organosulfur compounds & Stretch & Allin, allicin and di-allyl di-sulfide \\
674.58 & $\mathrm{C}=\mathrm{C}$ & Alkene & Bending & Allyl sulfides \\
\hline
\end{tabular}

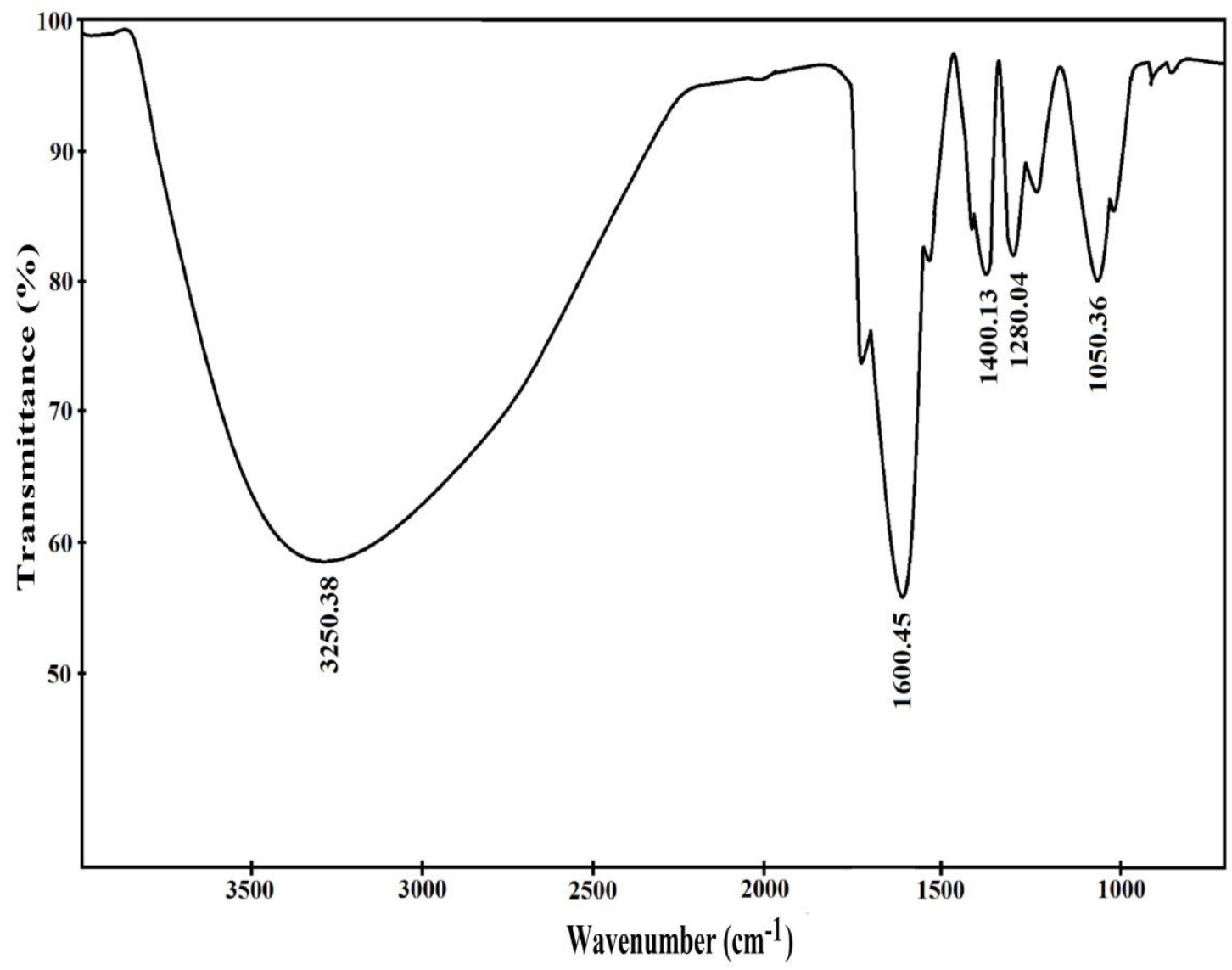

Fig. 5 FT-IR spectrum of solid mass obtained from evaporation of mint extract.

Table 5 Analysis of functional group, vibrational transitions and type of bonds found in the mint extract (Fourier transform infrared spectroscopy).

\begin{tabular}{lllll}
\hline Frequency $\left(\mathrm{cm}^{-1}\right)$ & Bond & Functional group & Type of bond & Specific type of bond \\
\hline $3,250.38$ & $\mathrm{O}-\mathrm{H}$ & Hydroxyl & Broad, stretch & Alcohol and phenolic compounds \\
$1,600.45$ & $\mathrm{C}=\mathrm{O}$ & Carbonyl & Stretch & Carbonyl compounds \\
$1,400.13$ & $\mathrm{C}-\mathrm{N}$ & Amine & Stretch & Aromatic amines \\
$1,280.04$ & $\mathrm{C}-\mathrm{N}$ & Amine & Stretch & Aromatic amines \\
$1,050.36$ & $\mathrm{C}-\mathrm{OH}$ & Primary alcohol & Stretch & Enzymes \\
\hline
\end{tabular}




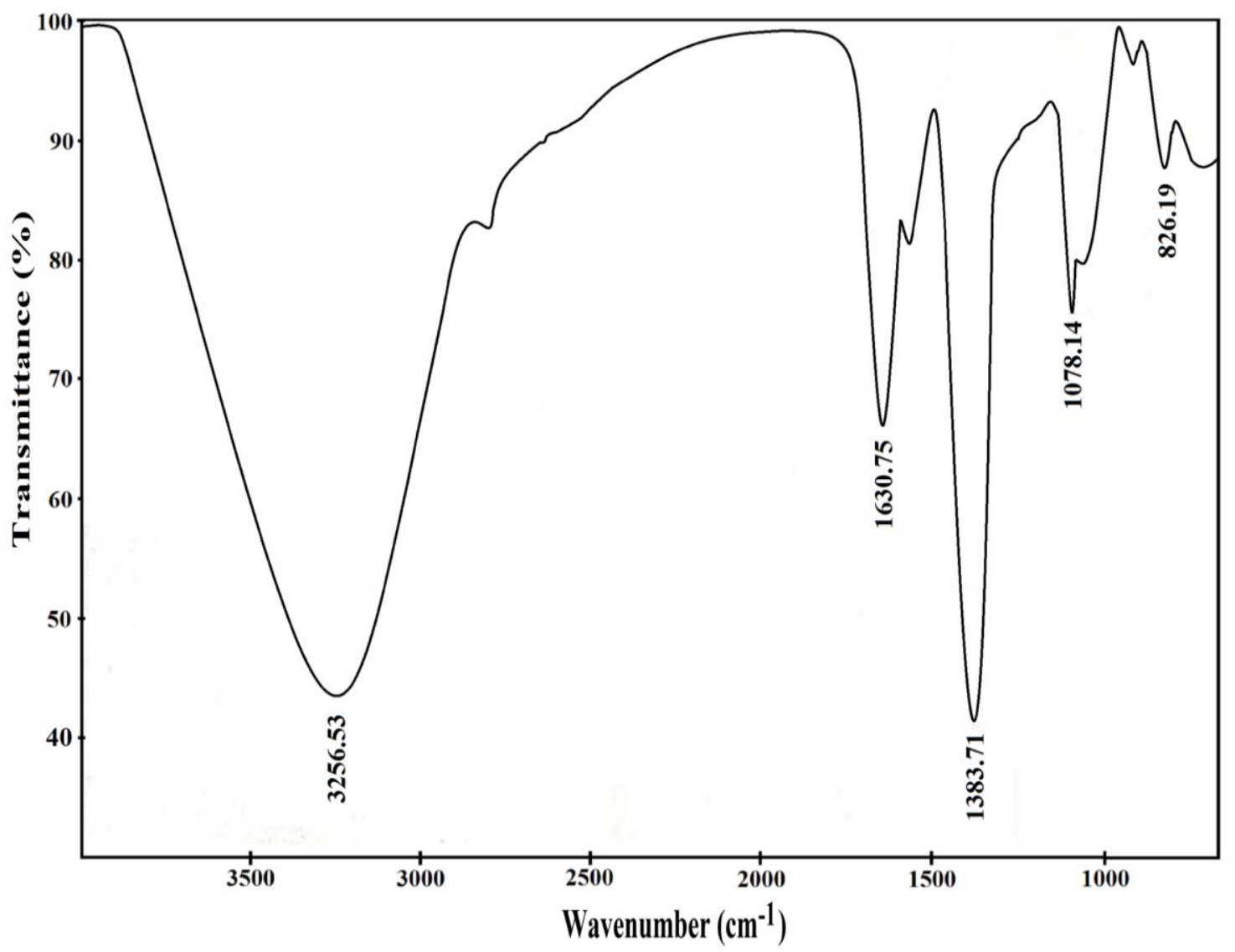

Fig. 6 FT-IR spectrum of solid mass obtained from evaporation of edible mushroom extract.

Table 6 Analysis of functional group, vibrational transitions and type of bonds found in the edible mushroom extract (Fourier transform infrared spectroscopy).

\begin{tabular}{lllll}
\hline Frequency $\left(\mathrm{cm}^{-1}\right)$ & Bond & Functional group & Type of bond & Specific type of bond \\
\hline $3,256.53$ & $\mathrm{O}-\mathrm{H}$ & Hydroxyl & Broad, stretch & Alcohol and phenolic compounds \\
$1,630.75$ & $\mathrm{C}=\mathrm{O}$ & Amide & Stretch & Enzymes \\
$1,383.71$ & $\mathrm{~S}=\mathrm{O}$ & Organosulfur compounds & Stretch & Sulfur compounds \\
$1,078.14$ & $\mathrm{C}-\mathrm{O}$ & Carbonyl & Stretch & Ester compounds \\
826.19 & $\mathrm{C}=\mathrm{C}$ & Alkene & Bending & Antioxidants \\
\hline
\end{tabular}

\subsection{The Effective Parameters on Electrospinning}

Process

Electrospinning technique is known as one of the most diverse methods for making nanofibers via electrostatic. Many factors and components are effective on the electrospinning process, and these components can combine into hundreds of different modes. The components were studied in four groups including: components of raw materials, components of electrospinning solution properties, components of process conditions and components of environmental factors. Each of components has subcategories that cause a large variety in the production of fibrous nanostructures. It should be noted that due to the wide range of materials and conditions, the conditions for the change of these components cannot be considered as a fixed and unchanged law, because each of these components can be significantly influenced by other components at a significance level [30].

\subsubsection{Structure and Properties of Polymer Material}

$\frac{4}{5}$ of honey weight is composed of carbohydrate, and the rest is composed of proteins, mineral salts, aroma 
compound, enzymes, vitamins, pollen and a small amount of water. The electrospinning behavior of any polymer material depends on its basic chemical structure, and they affect the applications of that material, primarily. For example, biocompatible polymer materials play a direct role in biocompatibility of nanofibers. Molecular weight: concentration and viscosity are the function of the original chemical structure of the polymer. In this paper, honey polymer material was used to produce nanofibers. The chemical composition of honey is diverse and complex.

\subsubsection{Properties of Electrospinning Solution}

By increasing the viscosity, the number of beads decreases. The absolute viscosity (dynamic or shear viscosity) of honey was measured by an Ostwald viscometer at a laboratory temperature $\left(20^{\circ} \mathrm{C}\right)$ and was calculated to be approximately $10,000 \mathrm{gr} / \mathrm{cm} \cdot \mathrm{sec}$ or 10,000 cps. Therefore, the momentum diffusivity (kinematic viscosity) was approximately equal to 80 $\mathrm{cm}^{2} / \mathrm{sec}$ or $7092 \mathrm{cst}$.

The role of solvent in electrospinning is effective and very important. It affects the electrospining process from different aspects. The nanofibers morphology is directly related to the solvents used. In this work, ethanol solvent was used to produce nanofibers. Also, in order to carry out the ventilation process in the electrospinning machine, the solvent exits from the conducive metal cage by a fan with the ability to operate time scheduling.

The amount of surface tension of honey is low and, as it was mentioned earlier, it is used as a suitable moisture absorbing material in making make-up products. Of course, the type of geographic area where honey is produced can affect its surface tension. Existence of surface tensile property leads to high adhesion to honey, and if the surface tension of honey is lower than its desired limit, the honey loses its adhesion and fluid quickly.

The dielectric constant is an important physical parameter for measuring the relative permittivity of a dielectric material. The solvent dielectric constant has a significant effect on electrospinning. This parameter is a criterion for polymer solution polarity. By increasing the dielectric constant, fewer beads and fibers with smaller diameter are produced. The dielectric constant of ethanol as solvent used in this research work at room temperature $\left(25^{\circ} \mathrm{C}\right)$ is 24.3 .

\subsubsection{Process Conditions}

According to previous study [31], it has been shown that increasing the applied voltage will increase the diameter of the produced nanofibers. Researchers say this is due to the more injection of the polymer into electrospinning jet. Also, at a lower voltage, by decreasing jet acceleration and weakening of the electric field, the electrospinning jet flight time increases, which is desirable for smaller fibers formation. Therefore, the voltage close to critical voltage can be suitable for achieving thinner fibers. In most cases, a higher voltage causes greater stretching of the solution because of the greater columbic forces in the jet as well as a stronger electric field and these effects are effective in reducing the diameter of the fibers and help to evaporate faster solvent and dry the fibers. In practice, at higher voltages (higher than the critical voltage), there is more tendency to form seeds. By changing the voltage of the device, the most favorable voltage for the production of nonbead nanofibers with a size uniform distribution with a minimum of droplet was selected on a 20,000-volt aluminum rotary collector.

Flow rate or jet flow velocity is the rate of arrival of the polymer mixture into the pump in order to form a taylor cone. In ideal mode, the flow rate should match the transfer rate of solution from needle tip. Under these conditions, nanofibers are obtained long and with uniform diameters. When the flow rate increases, the fibers diameter or the size of the beads increases, accordingly. In general, lower flow rate is preferable because of more time spent on evaporation.

Injection systems in electrospinning machine (Three Side Lab ES model, manufacture in Nanoazma Company) used in this paper prepare the flow rates 
between $0.1-10 \mathrm{~mL} / \mathrm{h}$, independently. The electrospinning jet flow rate was selected equal to 1.4 $\mathrm{mL} / \mathrm{h}$ in order to produce optimum nanofibers with minimum drops and the most suitable spraying.

\subsection{The Main Characteristics of the Nano-fiber Layers}

\subsubsection{Morphology and Arrangement}

The average diameter of the fibers, the surface porosity and the layers of the nanofibers, the hydrophobicity of the nanofibers and their layers are dependent on their morphology and microstructure. In this work, the morphology of electrospinning nanofibers was studied by SEM and data on the size and distribution of cavities were analyzed.

The images of SEM obtained from electrospinning of honey polymer solution nanofibers show the smooth, continuous and non-bead nanofibers with an average diameter of $240 \mathrm{~nm}$. The images of the SEM microscope show that adding $5 \%$ of garlic-mint-mushroom extract with equal volumetric ratios to the honey polymer solution and performing the electrospinning process produces uniform and continuous and non-bead nanofibers with an average diameter of $110 \mathrm{~nm}$. Because nanofibers containing $10 \%$ and $15 \%$ of garlic-mint-mushroom extract with equal volumetric ratios produce non-uniform, multi-diagonal fibers with an average diameter of 140 and $170 \mathrm{~nm}$ and whit the presence of bead on the fibers. Therefore, they are undesirable in terms of morphology, and they were ignored in continue research (Fig. 7).

In order to investigate the stability of nanofibres scaffold in human body, the honey polymer nanofibers enriched with antibacterial herbal extracts were immersed in acetate buffer solution with $\mathrm{pH}=5.5$ at $37{ }^{\circ} \mathrm{C}$ for 24 hours. Then, the samples removed from the solution were placed at room temperature $\left(25^{\circ} \mathrm{C}\right)$ for 24 hours until completely dry. After that, changes in the structure of nanofibers scaffolds were investigated by SEM before and after than located in acetate buffer solution. The results of research showed that the samples containing the honey polymer nanofibers enriched with antibacterial herbal extracts, in the presence of acetate buffer solution, retained their structure after 24 hours, completely.

The stability of nanofiber scaffolds is due to the presence of honey and the incorporation of the molecules
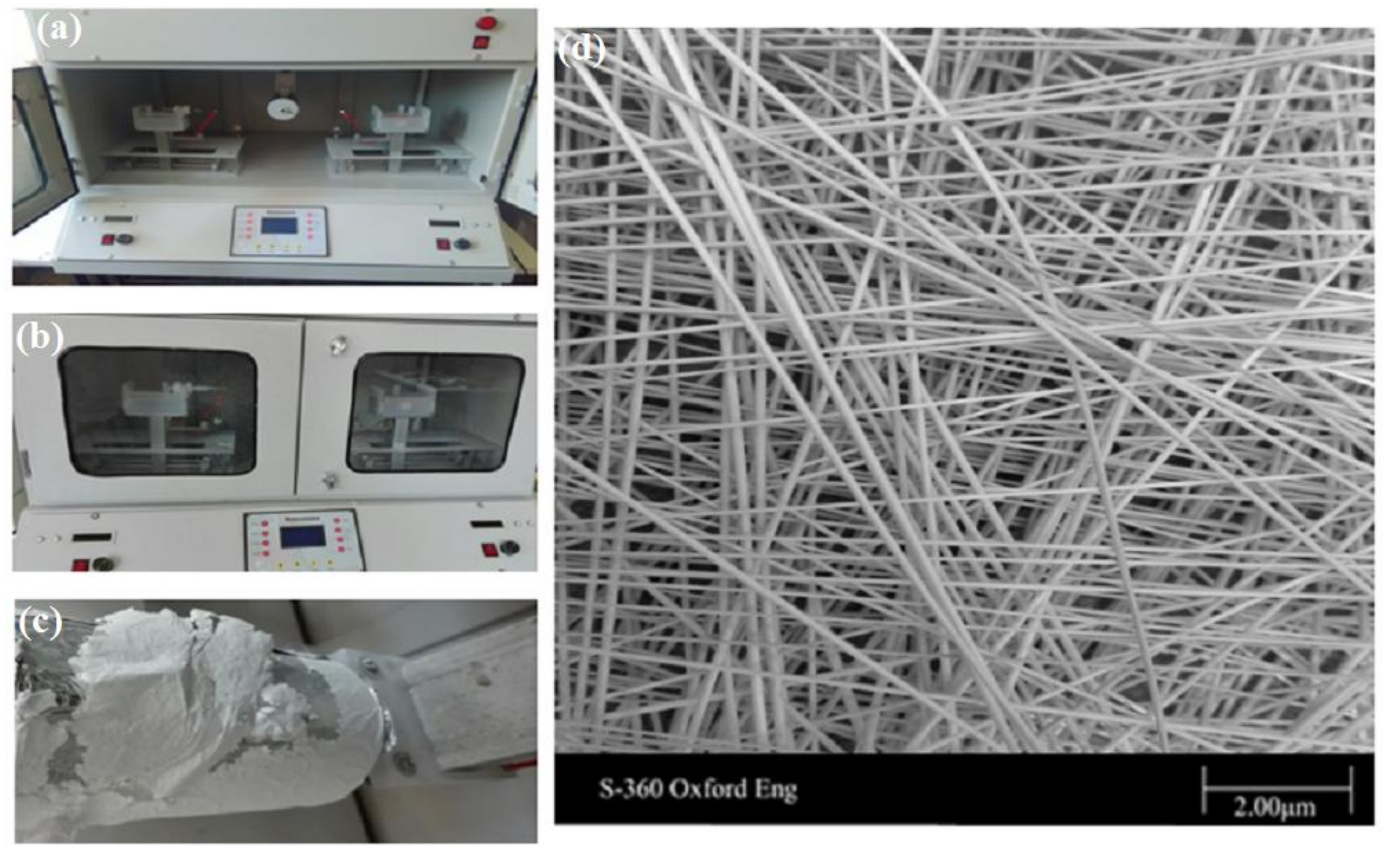

Fig. 7 Steps of electrospinning process: (a) The electrospinning device before doing the research work; (b) The electrospinning device after doing the research work; (c) Optimal sample; (d) SEM images of the electrospun nanofibrous polymeric (optimal sample). 

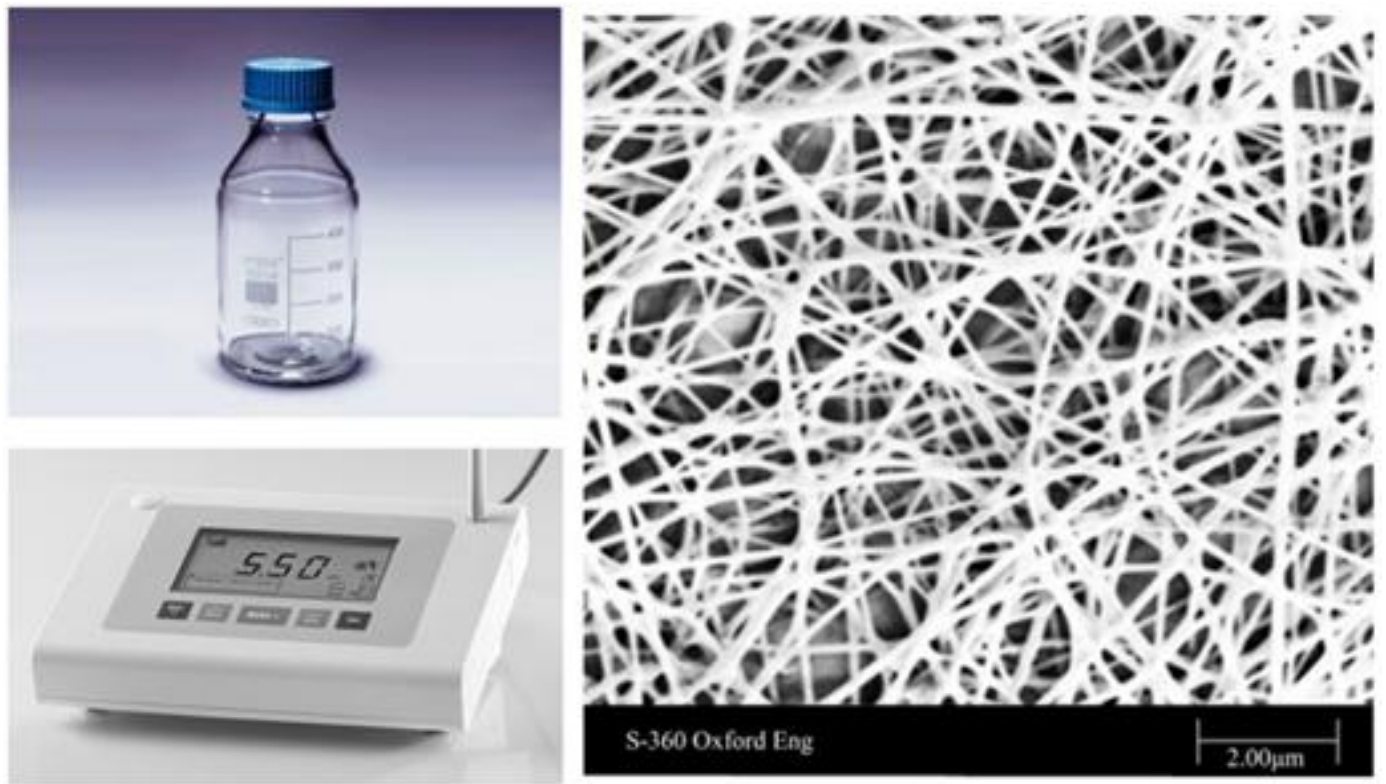

Fig. 8 SEM images of the electrospun nanofibrous polymeric (optimal sample) after staining in acetate buffer solution with pH $=5.5$ for 24 hours at $37^{\circ} \mathrm{C}$.

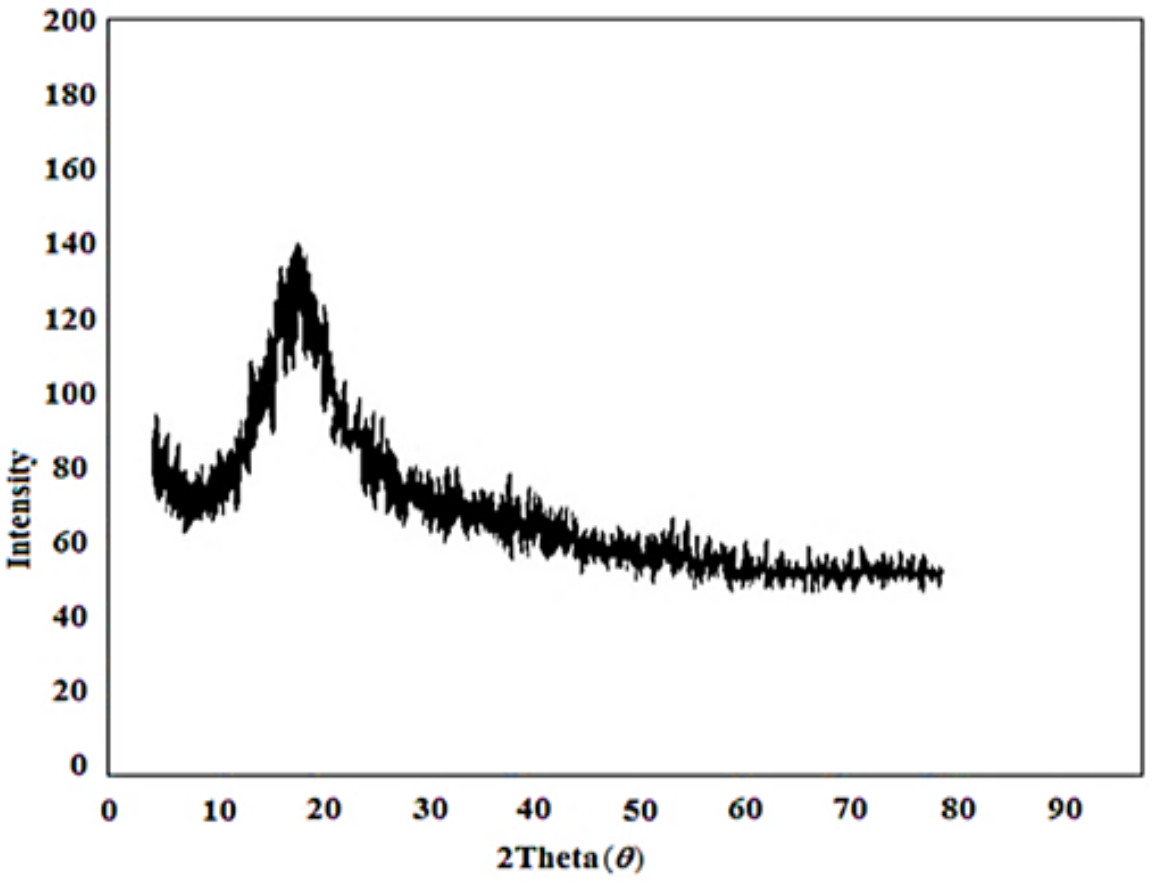

Fig. 9 The X-ray diffraction spectrum (XRD pattern) of the most desirable electrospun nanofibrous polymeric.

of this polymer. Investigation of water absorption by nanofibers scaffold showed that increasing the amount of water absorption and water content by adding antibacterial herbal extracts to honey polymer. By increasing the swelling rate of nanofiber scaffold, the antibacterial active ingredient is released faster than the nanofibers and into its release environment.
Increasing the swelling rate means that the nanofibers scaffold retains more moisture. As a result, at all stages of tissue repair, the discussed surface is somewhat moist. This will cause that the nanofibres scaffold does not stick to the wound, and when it is removed from the surface of the wound, it does not cause a patient's discomfort, and oxygen transit on it is 
done easily. In this way, the rate of repair and regeneration of the skin and bone tissue of damaged is increased (Fig. 8).

\subsubsection{Molecular Structure}

The molecular structure of nanofibers is effective on the mechanical, optical and thermal behavior of its layers. Chemical operations of nanofibres after electrospinning create favorable changes in the chemical structure of polymer molecules. In this research, the crystalline structure electrospun polymer nanofibres were investigated of X-ray diffraction spectroscopy (XRD). XRD analysis was obtained for the most optimum electrospun nanofibers with amorphous structure and $2 \theta=19$ (Fig. 9).

\section{Conclusions}

A summary of the effects of parameters on the morphology of produced nanofibers by electrospinning process is provided as following:

- Viscosity plays an important role in beads formation, and these defects are reduced by increasing viscosity (kinematic viscosity $=80 \mathrm{~cm}^{2} / \mathrm{sec}$ ).

- The high voltage power supply should be sufficiently capable of being overcome the viscosity and surface tension of polymer solutions and create jet (Voltage 20,000 volts).

- The solvent vapor pressure, which determines the rate of evaporation, should be such that the solvent is evaporated completely during the time that the fibers reach to the collector and removed from the polymer (ethanol).

- The flow rate should be equal to the transfer rate of solution from needle for establishing mass balance. In this condition, charges on the jet increase with increasing flow rate (flow rate $=1.4 \mathrm{~mL} / \mathrm{h}$ ).

- The fibers that accumulate on the surface of the insulator, often have a lower density than those that accumulate on the conductor surface (at ambient temperature).

- Reducing the diameter of the needle aperture to a certain value can reduce the diameter of the fiber (needle number $=20$ ). The distance between the nozzle and the collector plate should be such that provided possibility of complete evaporation of the solvent before being placed on the collector (15 centimeters).

- Optimum pressure and temperature should be used to produce the fibers with appropriate morphology (ambient pressure and temperature).

\section{References}

[1] Weijie, L. et al. 2017. "Structural Modulation of Gut Microbiota during Alleviation of Antibiotic-associated Diarrhea with Herbal Formula." Int J Biol Macromol 105: 1622-9.

[2] Lorna, M. et al. 2016. "Antimicrobial Resistance and Synergy in Herbal Medicine." J Herb Med 6: 53-8.

[3] Pauline, M. et al. 2016. "Honey: A Therapeutic Agent for Disorders of the Skin." J Microbiol Immunol 49: 161-7.

[4] Anitha, S. et al. 2017. Electrospun Materials for Tissue Engineering and Biomedical Applications. Woodhead Publishing.

[5] Heyu, L. et al. 2018. "Microsuspension of Fatty Acid Esters of Entecavir for Parenteral Sustained Delivery." Int J Pharm 543: 52-9.

[6] Vandamme, L. et al. 2013. "Honey in Modern Wound Care: A Systematic Review." Burns 39: 1514-25.

[7] Natália, M. et al. 2016. "Chemical Composition and Bioactive Compounds of Garlic (Allium sativum L.) as Affected by Pre- and Post-harvest Conditions: A Review." Food Chem 211: 41-50.

[8] Yanjun, Z. et al. 2017. "Modification of Garlic Peel by Nitric Acid and Its Application as a Novel Adsorbent for Solid-Phase Extraction of Quinolone Antibiotics." Chem Eng J 326: 745-55.

[9] Jianhua, Y., and Zhongjie, F. 2011. "Meta-analysis of the Effect of Garlic on Blood Pressure." Int J Cardiol 152: S84.

[10] John, A. M. 2011. "Occurrence and Biological Control of Postharvest Decay in Onion Caused by Fungi." J Nutr 131: 1027-31.

[11] John, A. M. 2006. "Preclinical Perspectives on Garlic and Cancer." J Nutr 136: 827-31.

[12] Om, P. et al. 2016. Essential Oils in Food Preservation, Flavor and Safety. Elsevier.

[13] Doaa, M. H. et al. 2017. "Biophenols of Mints: Antioxidant, Acetylcholinesterase, Butyrylcholinesterase and Histone Deacetylase Inhibition Activities Targeting Alzheimer's Disease Treatment." J Funct Foods 33: 345-62.

[14] Jingyan, W. et al. 2014. "Percutaneous Penetration Enhancement Effect of Essential Oil of Mint (Mentha 
haplocalyx Briq.) on Chinese Herbal Components with Different Lipophilicity." J Tradit Chin Med 1: 109-19.

[15] Behvar, A. et al. 2018. "Amylase, Glucosidase, Tyrosinase, and Cholinesterases Inhibitory, Antioxidant Effects, and GC-MS Analysis of Wild Mint (Mentha longifolia var. calliantha) Essential Oil: A Natural Remedy." Eur J Integr Med 22: 44-9.

[16] Kexin, Z. et al. 2018. "Recent Advances in Quality Preservation of Postharvest Mushrooms (Agaricus bisporus): A Review." Trends Food Sci Tech 78: 72-82.

[17] Yingyin, X. et al. 2016. "Effect of Plasma Activated Water on the Postharvest Quality of Button Mushrooms, Agaricus bisporus." Food Chem 197: 436-44.

[18] Jun, L. et al. 2013. "In Vitro and in Vivo Antioxidant Activity of Ethanolic Extract of white Button Mushroom (Agaricus bisporus)." Food Chem Toxicol 51: 310-6.

[19] Ganesan, D., and Sankaran, M. 2013. "A Detail Study of Phytochemical Screening, Antioxidant Potential and Acute Toxicity of Agaricus bisporus Extract and Its Chitosan Loaded Nanoparticles." J Pharm Res 6: 818-22.

[20] Koji, K. et al. 2017. "Novel Assay of Antibacterial Components in Manuka Honey Using Lucigenin-Chemiluminescence-HPLC." Anal Chim Acta 954: 151-8.

[21] Javad, T., and Youhong, T. 2017. "Honey/PVA Hybrid Wound Dressings with Controlled Release of Antibiotics: Structural, Physico-Mechanical and In-vitro Biomedical Studies." Mat Sci Eng C 77: 318-25.

[22] Rufat, A. et al. 2016. "Antioxidant (AO) and Free Radical Quenching (FRQ) Activities of Traditional Medicine
Substances, Honey and Vinery Products of Azerbaijan Republic.” J Biotechnol 231: S61.

[23] Muhammad, Q. K. et al. 2017. "Fabrication and Characterization of Nanofibers of Honey/Poly (1, 4 Cyclohexane Dimethylene Isosorbide Trephthalate) by Electrospinning." Mat Sci Eng C 81: 247-51.

[24] Gabriel, L. C. et al. 2016. "Development of Meniscus Substitutes Using a Mixture of Biocompatible Polymers and Extra Cellular Matrix Components by Electrospinning.” Mat Sci Eng C 61: 893-905.

[25] Mostafa, F., and Gelareh, K. 2014. "Investigation of Biodiesel Production Using Modified Strontium Nanocatalysts Supported on the ZSM-5 Zeolite." Ind Crop Prod 58: 298-304.

[26] Mostafa, F., and Esmaeil, S. 2015. "Catalytic Performance and Characterization of $\mathrm{Cs}-\mathrm{Ca} / \mathrm{SiO}_{2}-\mathrm{TiO}_{2}$ Nanocatalysts for Biodiesel Production." J Mol Catal A-Chem 404: 131-8.

[27] Vineet, S. et al. 2012. "Garlic: A Concise Drug Review with Probable Clinical Uses.” Int J Drug Dev Res 4: 9-17.

[28] Diane, M., and Jeffrey, B. B. 2006. "A Review of the Bioactivity and Potential Health Benefits of Peppermint Tea (Mentha piperita L.).” Phytother Res 20: 619-33.

[29] Bożena, M. et al. 2018. "Anti-inflammatory Properties of Edible Mushrooms: A Review.” Food Chem 243: 373-81.

[30] Luciana, M. et al. 2010. "Electrospinning from Room Temperature Ionic Liquids for Biopolymer Fiber Formation." Green Chem 12: 1883-92.

[31] Brendan, R., and Barry, L. 2011. Electrospinning for Tissue Regeneration. Elsevier. 